\title{
Pressure characteristics of pneumatic driven microfluidic chip system adopting a stepper motor
}

\author{
Yunfeng Zhu ${ }^{1, a}$, Jinxian Wang ${ }^{1, b}$,Songjing $\mathrm{Li}^{1, \mathrm{C}^{*}}$ \\ ${ }^{1}$ Department of Fluid Control and Automation, Harbin Institute of Technology, Harbin,150001, China \\ azhuyunfeng1988@126.com, b dao9413@163.com, 'lisongjing@hit.edu.cn
}

\begin{abstract}
Keywords: Pneumatic microfluidic chip system, Stepper motor, Pressure characteristic, Simulink Abstract. A pneumatic driven microfluidic chip system adopting a stepper motor is presented in this paper which changing the valve opening with a stepper motor and controlling the pressure of the gas cavity. The simulation model was built by using Simulink nonlinear module after expounding the structure and working principle and establishing the mathematical model of the system. The pressure dynamic response characteristics of the pneumatic driven microfluidic chip system adopting a stepper motor on different valve opening were given based on simulation. The pressure characteristics test curves of the gas container were obtained and compared with the simulation results by building a test platform.
\end{abstract}

\section{Introduction}

Microfluidic chip is a kind of technology that integrates thousands of micro-flow channels on several square centimeters chip. Microfluidic chip is also called lab-on-a-chip because it implements the functions on the biochemical field such as sample preparation, process reaction, separation, detection, etc[1]. It is praised as one of the seven techniques changing the world by Business 2.0[2]. All kinds of experiments can be completed effectively with very small amounts of reagents[3], instead of the conventional biochemical experiment platform[4]. PDMS and organic glass is used to encapsulation by the way of soft etching because it is convenience to manufacture, simple to control and easy to realize large scale integration[5]. PDMS membrane deformation between the pneumatic micro-flow channel and the liquid micro-flow channel is caused by pressure changes of pneumatic micro-flow channel, and it controls the on-off of the liquid micro-flow channel and the sample delivery. Team Quake has achieved a large scale integrated of thousands of micro-valves and hundreds of reactors on a microfluidic chip[6], which is an important technology breakthrough on the field of microfluidic chip.

At present, there are two types of fluid control technology of the microfluidic system. One of them is driven by fluid outside the microfluidic chip, controlling the flow of microfluidic chip directly by the external flow equipment. It includes peristaltic pump, injection pump pressure driven flow equipment, and was widely used for high flow control accuracy, but it is difficult to achieve miniaturization with a large volume[7]. Another one is driven by fluid onside the microfluidic chip, integrating microvalve or micropump on the microfluidic chip, such as multi valve coordination control, micro piezoelectric pump, micro peristaltic pump, micro electroosmosis pump, etc. It realizes flow driven by controlling opening of the valve, switching frequency and switching sequence[8]. This kind of flow driven equipment is possibly integrated with the microfluidic chip, but the flow control accuracy is not enough, the same with the flow adjustment range[9].

With the development and widely use of microfluidic chip, the request of miniaturization, integration and economical is higher. For the requirements of the Pneumatic microfluidic chip control system, this article put forward a pneumatic microfluidic chip system driven by a stepper motor based on the principle of pressure driven liquid flow rate, combining good elasticity of PDMS with displacement characteristics and control precision of the stepper motor[10], and studied the pressure characteristics. By controlling the valve opening with a stepper motor, the system has high control accuracy and wide control range. At the same time, with the rapid prototyping technology of 
microfluidic chip production, the overall system volume has been reduced greatly. So, it is easy to achieve the integration and miniaturization of microfluidic chip and the external devices in the future.

\section{Structure and Working Principle}

Pneumatic microfluidic chip system driven by a stepper motor consists of gas source, micro valve, stepper motor, pipes, gas-liquid interaction device, sensor, control circuit, etc. Control object is microfluidic chip, and the principle is shown in figure 1. Firstly, gas get through the valve into gas-liquid interaction device, and then the valve opening changes by controlling the stepper motor drives so that gas in closed container could send the liquid filling the microfluidic chips. At last, the liquid will flow into the waste liquid pool.

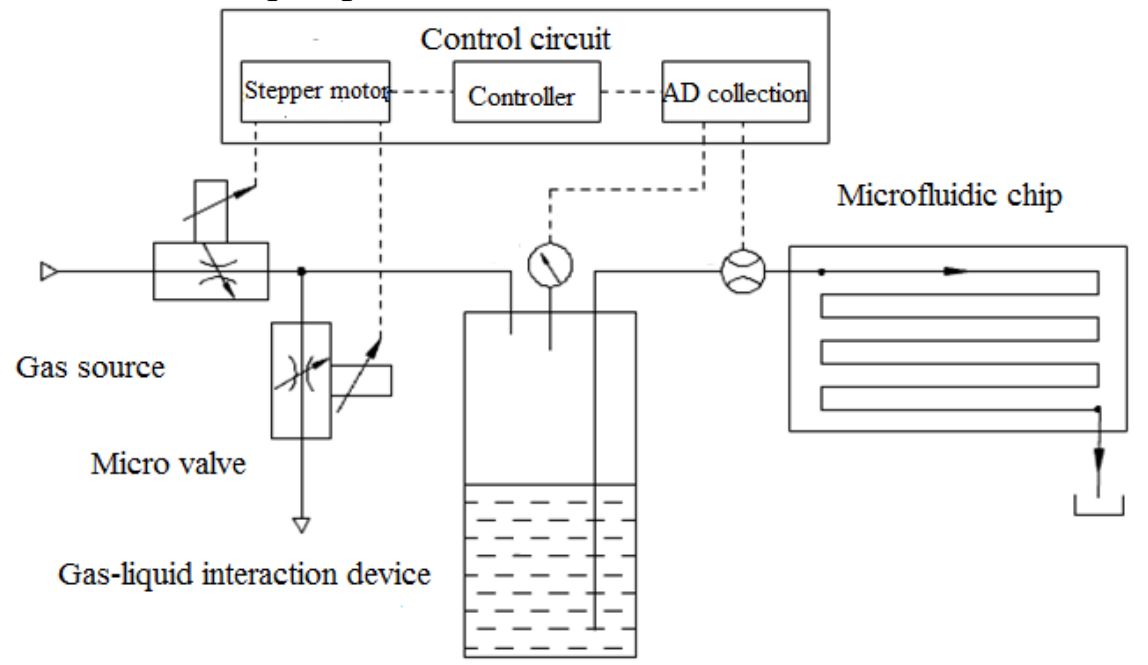

Fig. 1 Working principle of pneumatic microfluidic chip system

The structure of micro valve is shown in Fig. 2. Screw nut and slide valve core are fixed, and they move in the direction of the stepper motor shaft with the effect of baffle, directly affecting the PDMS flow channels and changing the valve opening.

a)

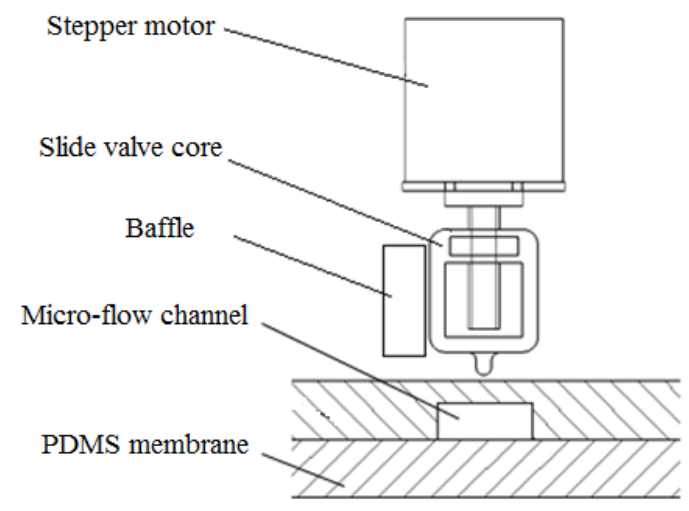

b)

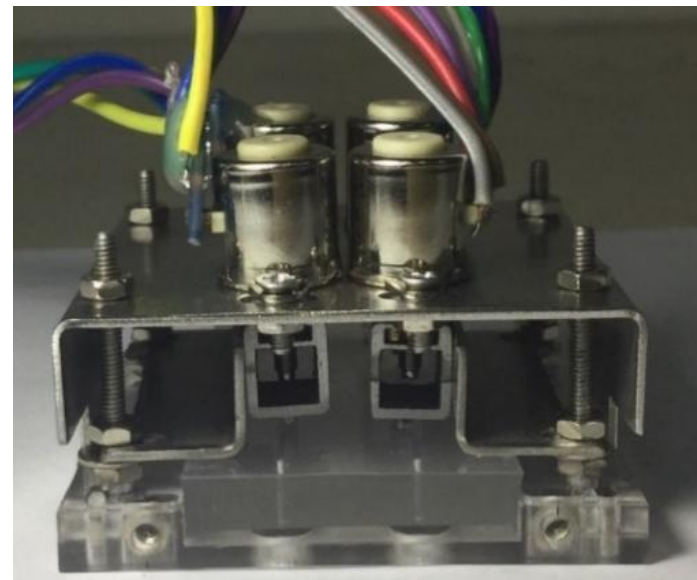

Fig. 2 Structure of the micro valve $\mid$ a). Structure of the micro valve driven by a stepper motor; $b$ ). The entity of pneumatic stepper-motor micro valve.

\section{Simulation}

Mathematic Model. Gas flow process of the micro valve is very complex, and the mass flow equation of a convergent nozzle was used this paper. According to Sanville flow formula, the mass flow rate of air inlet micro valve and air outlet micro valve could be listed as: 


$$
\begin{aligned}
& q_{1}= \begin{cases}\left.A_{1} p_{s} \sqrt{\frac{k}{R T_{s}} \cdot \frac{2}{k-1}} \cdot \sqrt{\left(\left(\frac{p}{p_{s}}\right)^{\frac{2}{k}}-\left(\frac{p}{p_{s}}\right)^{\frac{k+1}{k}}\right.}\right) & b \leq \frac{p}{p_{s}} \leq 1 \\
A_{1} p_{s} \sqrt{\frac{2 k}{R T_{s}(k+1)}} \cdot\left(\frac{2}{k+1}\right)^{\frac{1}{k-1}} & 0 \leq \frac{p}{p_{s}}<b\end{cases} \\
& q_{2}= \begin{cases}\left.A_{2} p \sqrt{\frac{k}{R T} \cdot \frac{2}{k-1}} \cdot \sqrt{\left(\frac{p_{0}}{p}\right)^{\frac{2}{k}}-\left(\frac{p_{0}}{p}\right)^{\frac{k+1}{k}}}\right) & b \leq \frac{p_{0}}{p} \leq 1 \\
A_{2} p \sqrt{\frac{2 k}{R T(k+1)}} \cdot\left(\frac{2}{k+1}\right)^{\frac{1}{k-1}} & 0 \leq \frac{p_{0}}{p}<b\end{cases}
\end{aligned}
$$

Where $A_{1}$ and $A_{2}$ are respectively the orifice areas of the air inlet micro valve and air outlet micro valve, $T_{s}$ is the temperature of the air source, $T$ is temperature of the gas container, $p$ is the pressure of the gas container, $p_{s}$ is the pressure of the gas source, and $R$ is the isentropic exponent of the gas.

The pressure changes of the gas container can be obtained with the mass conservation and perfect gas equation of state:

$$
\frac{d p}{d t}=\frac{k}{V_{0}+\frac{1}{4} \pi D^{2} x} \cdot\left(R T \cdot\left(q_{1}-q_{2}\right)-p \cdot \frac{1}{4} \pi D^{2} \cdot \frac{d x}{d t}\right)
$$

Where $x$ is the height of the volume change, $D$ is the diameter of the gas container, and $V_{0}$ is the volume of gas container when time is $t_{0}$.

Simulation Model Building. The simulation model was built by using Simulink nonlinear module. According to the mathematical model, the simulation model was built and shown in Fig. 3.

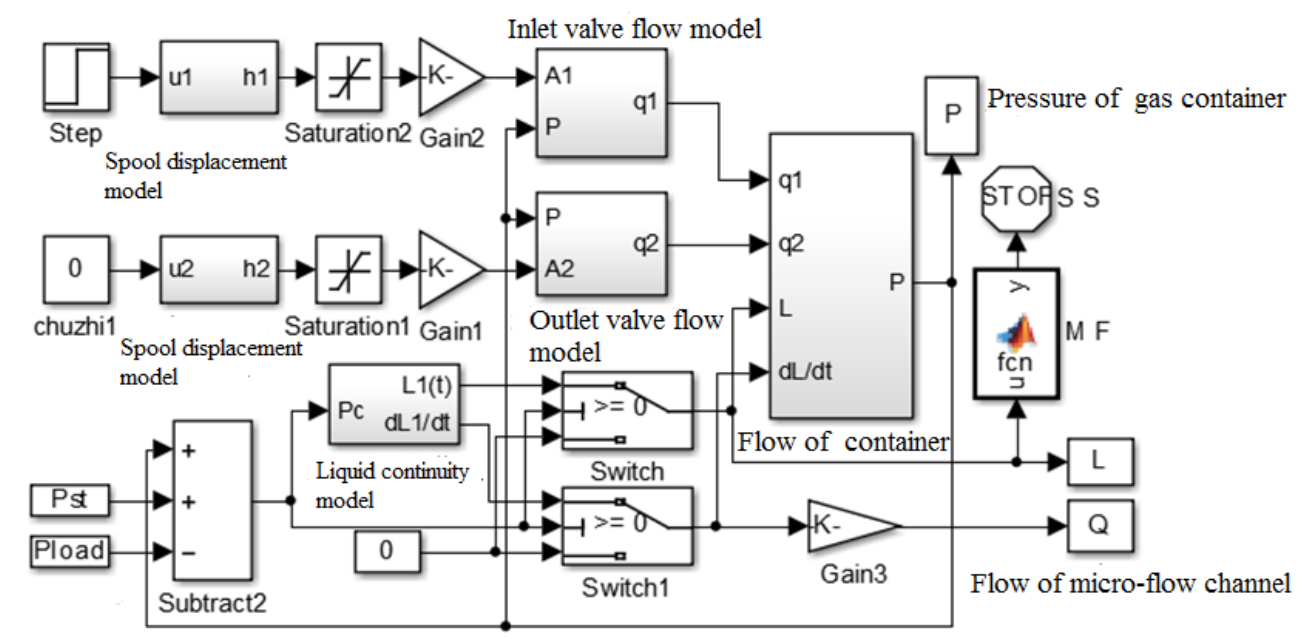

Fig. 3 Simulation model of pneumatic microfluidic chip system driven by a stepper motor

Simulation Analysis. According to the fluid model, open loop simulation model of pneumatic microfluidic chip system was established. The pressure of the gas source was set as $0.15 \mathrm{MPa}$, working medium was set as deionized water, filling flow channel of microfluidic chip was set as rectangular straight channel, and the height and width of the channel was respectively $50 \mu \mathrm{m}$ and $100 \mu \mathrm{m}$.

In the simulation of pressure dynamic characteristics of pneumatic microfluidic chip system, the valve opening step signal was respectively set as 0.6 times, 0.8 times and 1 times, and the simulation results are shown in Fig. 4. 


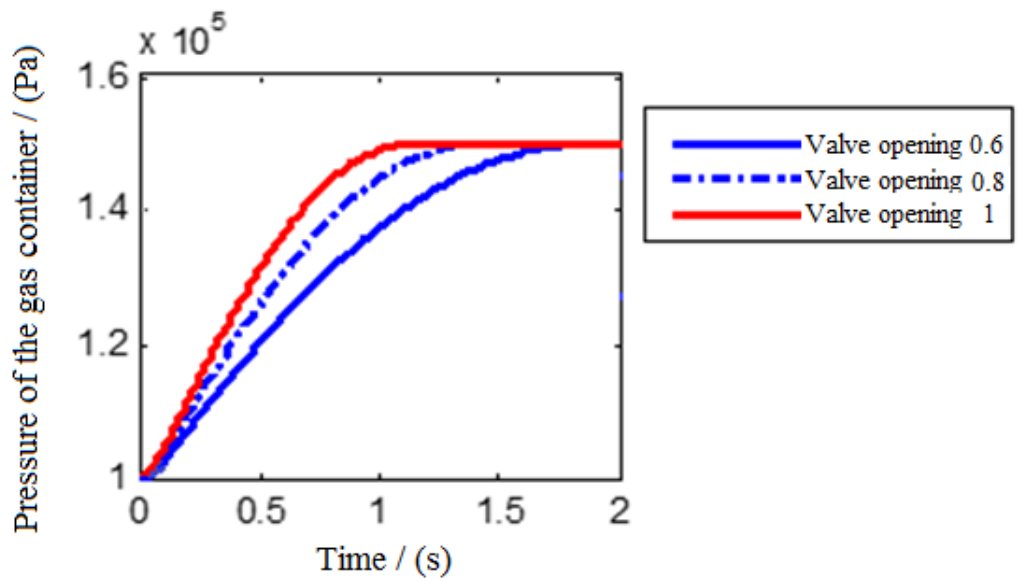

Fig. 4 Pressure characteristics of different valve opening

The simulation results show that the response time of the gas pressure to reach steady state is different due to the valve opening step signal. As the valve opening increasing, the pressure change rate of the gas container increases, and the steady pressure will reach steady state in a shorter time. When the valve opening is up to $60 \%$, the dynamic response time is about $1.7 \mathrm{~s}$. When the valve opening is up to $100 \%$, the dynamic response time is about $1.1 \mathrm{~s}$.

\section{Experiments}

Experiment platform was built with pressure control module, gas-liquid interaction device, microfluidic chip, gas source and regulating elements based on the structure principle of pneumatic microfluidic chip system, as shown in Fig. 5. Signal of the gas pressure sensor was collected through Advantech PCI-1710, recording the curves of the gas pressure in the computer.

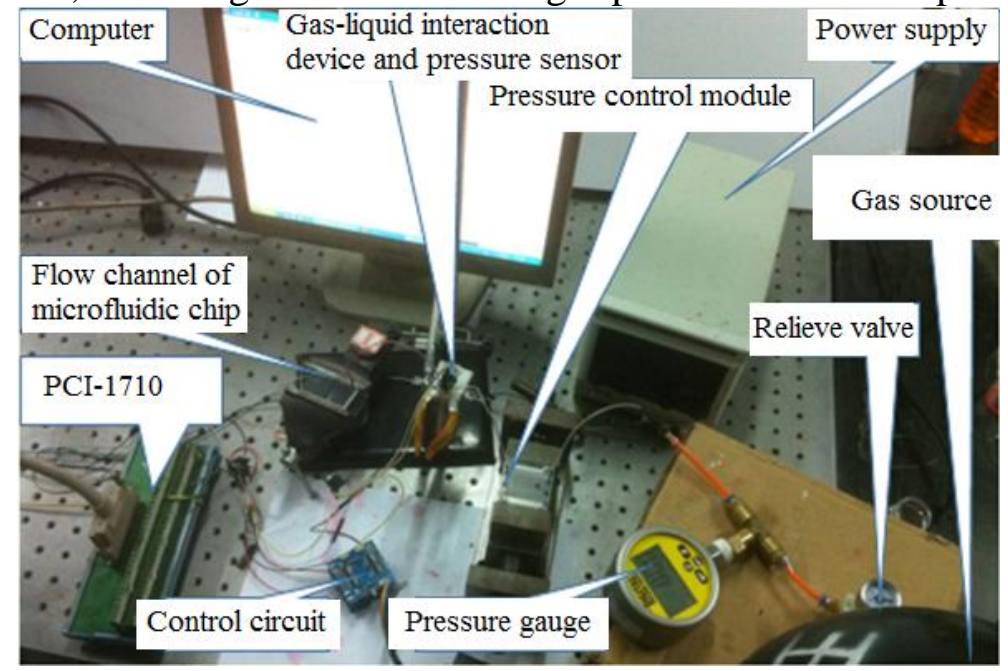

Fig. 5 Pressure characteristics experiments of pneumatic microfluidic chip system

Valve opening step response changes when the stepper motor driver input different step signal, and height of the flow channel changes from 0 to $30 \mu \mathrm{m}, 40 \mu \mathrm{m}$ and $50 \mu \mathrm{m}$ as well. The gas pressure sensor detected the pressure of the gas container, collected through Advantech PCI-1710 and save the data in computer in the process. Pressure characteristics of pneumatic microfluidic chip system were shown in Fig. 6 . When the valve opening is up to $60 \%$, the dynamic response time is about $1.7 \mathrm{~s}$ and the steady pressure is $0.148 \mathrm{MPa}$. When the valve opening is up to $100 \%$, the dynamic response time is about $1.1 \mathrm{~s}$ and the steady pressure is $0.15 \mathrm{MPa}$. It is obviously that the experimental results agree well with the simulation results. 


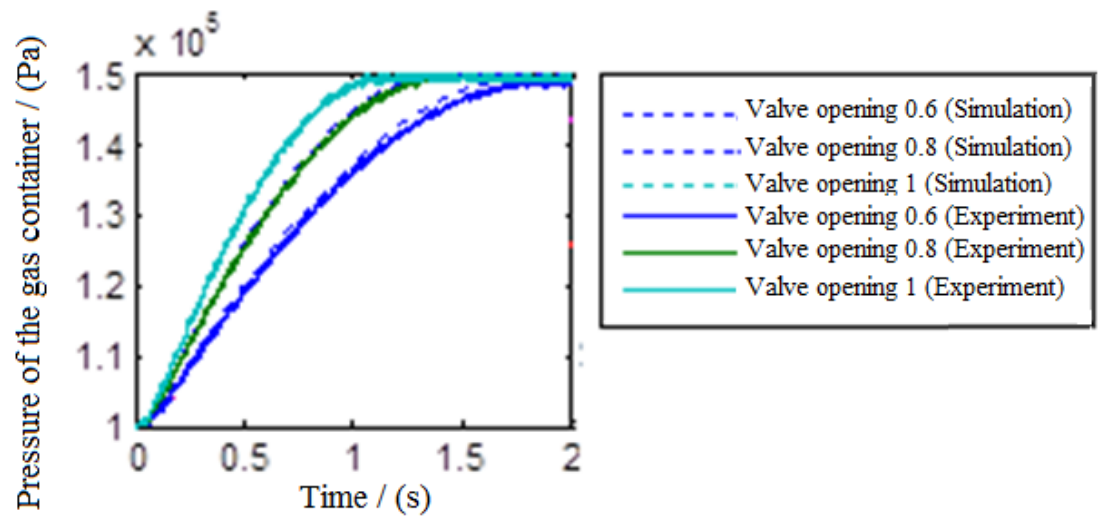

Fig. 6 Pressure characteristic curves of pneumatic microfluidic chip system

\section{Summary}

Pressure characteristics of pneumatic microfluidic chip system on different valve opening step signal by experiment are basically the same with the simulation results. When the valve opening is small, the pressure of the gas container changes gently. When the valve opening is larger, the pressure of the gas container rises more quickly, and slope of the rising becomes slower gradually on the effect of pneumatic impact. The pressure of the gas container in the experiment changes slightly slower than the simulation results, especially when the valve opening is small. The main reason may be that there is a leak in the system pipeline, and sometimes pressure loss at the pipe joint. The influence of the leakage and pressure loss on the pressure of the gas container will decrease as the valve opening increases.

\section{References}

[1] B.C. Lin \& J.H. Qin: Lab on a chip. Chinese Journal of Chromatography, 2005, 23(5): 456-463.

[2] Berthier J Microdrops and digital microfluidics[M] William Andrew, 2012.

[3] Janasek D, Franzke J, Manz A. Scaling and the design of miniaturized chemical-analysis systems[J]. Nature, 2006, 442(7101): 374-380.

[4] Balagaddé F K, You L, Hansen C L, et al. Longterm monitoring of bacteria undergoing programmed population control in a microchemostat[J]. Science, 2005, 309(5731): 137-140.

[5] HONG J W, QUAKE S R. Integrated nanoliter systems [J] . Nature Biotechnology, 2003, 21( 10): :1179-1183.

[6] T. Thorsen, S. J. Maerkl, S. R. Quake. Microfluidic large-scale integration. Science, 2002, 298(5593): 580-584

[7] Zhang P, S.Y. Wang, C.Y. Yu, et al. The Development Tendencies of Infusion Pumps I Syringe Pumps[J]. Chinese Journal of Medical Instrumentation, 2009, 33(4):282-285.

[8] Amirouche F, Zhou Y, Johnson T. Current micropump technologies and their biomedical applications[J]. Microsystem Technologies, 2009, 15(5):647-666.

[9] Hansen C L, Quake S R, Austin R H. From The Cover: Systematic investigation of protein phase behavior with a microfluidic formulator[J]. Proceedings of the National Academy of Sciences of the United States of America, 2004, 101(40):14431-6.

[10] Wang J X, Wen Z, Li S J. Design of a Pneumatic Flow Rate Control Microvalve Driven by a Stepper-Motor[J]. Applied Mechanics \& Materials, 2015, 779:244-249. 